\title{
Impacto de uma estratégia educativa na qualidade de vida e no autocuidado de pacientes com Insuficiência Cardíaca descompensada após alta hospitalar
}

Impact of an educational strategy on the quality of life and not self-care of patients with decompensated Heart Failure after discharge

Impacto de una estrategia educativa sobre la calidad de vida y el auto cuidado de pacientes con Insuficiencia Cardíaca descompensada tras el alta

Gustavo Venícius da Silva Santos ORCID: https://orcid.org/0000-0002-0463-7928 Universidade Tiradentes, Brasil

E-mail: gustavovinicius99@hotmail.com

Adão Renato de Jesus Freire ORCID: https://orcid.org/0000-0001-7166-2392 Universidade Tiradentes, Brasil E-mail: adao_jesus10@hotmail.com

Cleidinaldo Ribeiro de Góes Marques ORCID: https://orcid.org/0000-0003-2026-1112 Universidade Federal de Sergipe, Brasil E-mail: clei_ribeiro@hotmail.com

Deyse Mirelle Souza Santos

ORCID: https://orcid.org/0000-0001-8310-2448 Universidade Tiradentes, Brasil E-mail: deysemirellle@hotmail.com Aislayne Rodrigues Valentim ORCID: https://orcid.org/0000-0002-5629-9123 Universidade Tiradentes, Brasil E-mail: aislayne.rodrigues@gmail.com

Arthur César de Melo Tavares ORCID: https://orcid.org/0000-0002-3814-390X Universidade Tiradentes, Brasil

E-mail: arthur.melo.tavares@hotmail.com

Ana Liz Pereira de Matos

ORCID: https://orcid.org/0000-0003-4584-5827

Universidade Federal de Sergipe, Brasil E-mail: analizuneb@gmail.com

Yasmim Anayr Costa Ferrari

ORCID: https://orcid.org/0000-0003-1766-341X

Universidade Federal de Sergipe, Brasil

E-mail: yasmimanayr@hotmail.com

Maíra Ávila Fontes Trindade

ORCID: https://orcid.org/0000-0002-6268-3308

Universidade Federal de Sergipe, Brasil

E-mail: mairaftrindade@hotmail.com

Ana Caroline Araújo Temoteo

ORCID: https://orcid.org/0000-0003-1013-8954

Faculdade Dom Pedro II de Sergipe, Brasil E-mail: temoteoa35@gmail.com

Edna das Virgens Santana

ORCID: https://orcid.org/0000-0002-5731-6407 Universidade Federal de Sergipe, Brasil E-mail: ednasdc.enf@gmail.com

João Paulo Santos de Oliveira

ORCID: https://orcid.org/0000-0002-3099-8006

Faculdade Dom Pedro II de Sergipe, Brasil

E-mail: jopogovidanova@gmail.com

José Lucas Abreu Nascimento

ORCID: https://orcid.org/0000-0001-6538-6125

Faculdade Dom Pedro II de Sergipe, Brasil

E-mail: lucasabreu007@outlook.com 


\author{
Gabriela Silva Oliveira \\ ORCID: https://orcid.org/0000-0003-2180-5580 \\ Universidade Federal de Sergipe, Brasil \\ E-mail: gabiolivers2506@gmail.com \\ Eduesley Santana Santos \\ ORCID: https://orcid.org/0000-0001-8545-5677 \\ Universidade Federal de Sergipe, Brasil \\ E-mail: eduesley.santos@gmail.com
}

\begin{abstract}
Resumo
O presente estudo teve como objetivo avaliar o impacto de uma intervenção educativa no autocuidado e Qualidade de Vida (QV) de pacientes com Insuficiência Cardíaca (IC) descompensada após alta hospitalar. Trata-se do recorte de um Ensaio Clínico Randomizado, cuja amostra foi dividida em Grupo Intervenção (GI) e Grupo Controle (GC). O GI foi acompanhado e orientado por enfermeiros de forma presencial e telefônica, com realização de exame físico, aplicação de questionários (QV e autocuidado) e orientação terapêutica. Ambos os grupos foram avaliados no $60^{\circ}$ dia após alta. Os dados foram analisados por meio da ferramenta Statistical Package for Social Sciences, versão 21.0. Dos 22 pacientes avaliados, 15 foram do GC e 7 do GI. O GI apresentou melhores escores em comparação ao GC quanto ao autocuidado ( $100 \%$ vs $47 \%, \mathrm{p}=0,02)$ e QV (44\% vs $20 \%$, p=0,36). A intervenção de enfermagem realizada apresenta-se como estratégia factível para melhora do autocuidado e qualidade de vida dos pacientes com IC.
\end{abstract}

Palavras-chave: Insuficiência cardíaca; Autocuidado; Qualidade de vida; Enfermagem cardiovascular.

\begin{abstract}
This study aimed to evaluate the impact of an educational intervention on self-care and Quality of Life (QL) of patients with decompensated Heart Failure (HF) after hospital discharge. This is part of a Randomized Clinical Trial, whose sample was divided into Intervention Group (IG) and Control Group (CG). The IG was monitored and guided by nurses in person and over the phone, with a physical examination, application of questionnaires (QL and self-care) and therapeutic guidance. Both groups were evaluated on the 60th day after discharge. Data were analyzed using the Statistical Package for Social Sciences tool, version 21.0. Of the 22 patients evaluated, 15 were from the CG and 7 from the IG. The IG had better scores compared to the CG regarding self-care (100\% vs 47\%, $\mathrm{p}=0.02)$ and QoL (44\% vs $20 \%, \mathrm{p}=0.36$ ). The nursing intervention performed is presented as a feasible strategy to improve self-care and quality of life for patients with HF.
\end{abstract}

Keywords: Heart failure; Self-care; Quality of life; Cardiovascular nursing.

\title{
Resumen
}

Este estudio tuvo como objetivo evaluar el impacto de una intervención educativa sobre el autocuidado y la Calidad de Vida (CV) de pacientes con Insuficiencia Cardíaca (IC) descompensada tras el alta hospitalaria. Esto es parte de un Ensayo Clínico Aleatorizado, cuya muestra se dividió en Grupo de Intervención (GI) y Grupo de Control (GC). El GI fue monitoreado y guiado por enfermeras en persona y por teléfono, con examen físico, aplicación de cuestionarios (CV y autocuidado) y orientación terapéutica. Ambos grupos fueron evaluados el día 60 después del alta. Los datos se analizaron utilizando la herramienta Statistical Package for Social Sciences, versión 21.0. De los 22 pacientes evaluados, 15 eran del GC y 7 del GI. El GI tuvo mejores puntuaciones en comparación con el GC en cuanto al autocuidado (100\% vs $47 \%, \mathrm{p}=0,02)$ y $\mathrm{CdV}(44 \%$ vs $20 \%, \mathrm{p}=0,36)$. La intervención de enfermería realizada se presenta como una estrategia factible para mejorar el autocuidado y la calidad de vida de los pacientes con IC.

Palabras clave: Insuficiencia cardíaca; Autocuidado; Calidad de vida; Enfermería cardiovascular.

\section{Introdução}

Doenças cardiovasculares estão entre as maiores causas de morbimortalidade no Brasil e no mundo. Dentre elas, a Insuficiência Cardíaca (IC) se destaca pela sua alta expressividade, complexidade e gravidade. Na IC, as estruturas ventriculares do coração apresentam incapacidade de armazenar e ejetar o sangue recebido para suprir as demandas exigidas pelo organismo, uma incapacidade que pode ter como causa alterações anatômicas e/ou fisiológicas sofridas pelo órgão (Comitê Coordenador da Diretriz de Insuficiência Cardíaca, 2018; Boisvert et. al., 2015).

A IC configura-se como evento de repercussão mundial, bem como um problema epidêmico em progressão. Desde 2017, a incidência média anual é de um milhão de casos diagnosticados. Dados extraídos da American Heart Association, indicam que a doença vem sendo prevalente em aproximadamente 6,5 milhões de indivíduos nos Estados Unidos, com estimativa de aumento de 46\% até 2030 (Boisvert et. al., 2015; Bozkurt \& Khalaf, 2017). Na América Latina, a IC descompensada é a principal causa de hospitalizações cardíacas (Flores et. al., 2020). 
Por conseguinte, no Brasil, entre os anos de 2015-2020, o número de internações hospitalares pela IC já ultrapassou 1,2 milhão de casos (SIH/SUS) (Brasil, 2021). Na região Nordeste do país, registraram-se 273.814 pacientes internados por esta síndrome, destes, 5.365 foram notificados em Sergipe neste mesmo período. Dos 5.365 casos, computou-se um total de 1.052 óbitos pela IC neste estado (Brasil, 2021). Mediante tais números, observa-se a necessidade do desenvolvimento de estratégias que auxiliem os pacientes a identificarem seu estado de saúde e manejá-lo (Linn, Azzolin \& Souza, 2016).

Nesta perspectiva, autocuidado é definido como conjunto de ações desempenhadas pelas pessoas em seu cotidiano com o intuito de prevenir, controlar ou reduzir o impacto das condições de saúde das quais são portadoras (Boisvert et. al., 2015; Koirala et al., 2018; Conceição et. al., 2015). Entretanto, para que a prática do autocuidado seja cumprida, pacientes e seus cuidadores devem entender sobre as condições de saúde que portam. Deste modo, quando o autocuidado é negligenciado, podese resultar em piora dos sintomas, reinternações hospitalares e óbitos (Bozkurt \& Khalaf, 2017).

Paralelamente, a identificação e utilização de métodos para melhorar o autocuidado na IC pode levar a melhores resultados clínicos e consequentemente, melhor Qualidade de Vida (QV) (Creber et. al., 2016). A QV é definida como algo complexo, multifatorial, subjetivo e autoperceptível pelos indivíduos, que varia de acordo com os sistemas de valores nos quais estes estão inseridos e sofre grande influência de fatores culturais, socioeconômicos, genotípicos e fenotípicos (Who, 1995). Diante da volatilidade conceitual da QV, avaliá-la em portadores de IC torna-se uma tarefa difícil e necessária, pois estes demandam de terapias otimizadas e de elevado custo para os serviços de saúde (Carvalho et. al., 2020; Sousa et. al., 2017).

Os profissionais de Enfermagem são instrumentos essenciais para a manutenção da saúde consequente à ampla participação nos diversos níveis de atenção à saúde (MOTA et. al., 2020). Em virtude disso, diretrizes nacionais recomendam o acompanhamento de portadores de IC por estes profissionais, com ênfase no enfermeiro e em sua atuação por meio da elaboração de estratégias educativas para o gerenciamento domiciliar destes cardiopatas, que favoreçam a redução de reinternações e mortalidade (Comitê Coordenador da Diretriz de Insuficiência Cardíaca, 2018; Montera et. al., 2009)

Diante da escassez de estudos locais que avaliem o impacto de intervenção educativa liderada por enfermeiro no autocuidado e QV de pacientes com IC descompensada, o estudo foi realizado com a perspectiva de contribuir para assistência a estes pacientes com ênfase em configurações ambulatoriais. No intuito de proporcionar redução dos custos com a saúde, o risco de piores resultados em longo prazo e melhoria da qualidade dos cuidados com os pacientes. O presente estudo teve como objetivo avaliar o autocuidado e QV de pacientes com IC descompensada submetidos a uma intervenção educativa pós alta hospitalar.

\section{Metodologia}

Trata-se do recorte de um Ensaio Clínico Randomizado (ECR) e controlado, correspondente ao período de 2018 a 2020, cadastrado no Registro Brasileiro de Ensaios Clínicos sob o número RBR-8dymr8 e norteado pelo checklist CONSORT na sua execução (Dainesi \& Aligieri, 2005). A pesquisa foi realizada após aprovação do Comitê de Ética e Pesquisa da Universidade Federal de Sergipe (UFS) conforme resolução 466/12 do Conselho Nacional de Saúde (CNS) sob número de parecer 2.897.628 (CAEE 93856218.0.0000.5546, autorizado em 17/09/2018).

$\mathrm{O}$ estudo foi realizado em quatro centros hospitalares localizados em três municípios do Estado de Sergipe. Foram incluídos no estudo pacientes de ambos os sexos, com idade igual ou superior a 18 anos, com o diagnóstico médico de IC com disfunção sistólica (Fração de Ejeção $\leq 49 \%$ pelo ECO) e internados por descompensação do quadro clínico relacionada às alterações de parâmetros vitais e exames laboratoriais e/ou de imagem (Ex: Ecocardiograma, Eletrocardiograma, Raio x).

Foram excluídos os pacientes que não possuíam contato telefônico; com problemas visuais, auditivos e/ou de locomoção incapacitantes às atividades de vida diária; classificação 4 pela New York Heart Association (NYHA); que estivessem 
participando de outra pesquisa com mesma temática; internados para realização de procedimentos cirúrgicos não relacionado à IC; residissem em município com mais de 100 km de distância de um dos hospitais de alocação e com déficit cognitivo.

Para identificar os pacientes com condições cognitivas ideais foi utilizado o instrumento "Mini Exame do Estado Mental - MEEM" validado para o português (Lenardt et. al., 2009). Foram adotados neste estudo os seguintes pontos de corte: pacientes analfabetos tiveram que obter no mínimo 13 pontos; aqueles com "1 a 7 anos de escolaridade" mínimo de 18 pontos; e com "8 ou mais anos" pelo menos 26 pontos.

$\mathrm{O}$ recrutamento dos pacientes se deu a partir dos pesquisadores da equipe de execução do estudo, mediante visitas diárias aos setores de internação/enfermarias de cada instituição no turno da manhã e/ou tarde. Os pacientes incluídos foram submetidos à aplicação dos instrumentos de coleta de dados sociodemográficos e clínicos e do MEEM. Os dados foram obtidos mediante entrevista com paciente e/ou acompanhante associado à busca ativa em prontuário. Após definição de inclusão, foi aplicado o Termo de Consentimento Livre e Esclarecido (TCLE) e Termo de Sigilo Telefônico. Os participantes incluídos na pesquisa foram distribuídos entre os Grupo Controle (GC) e Grupo Intervenção (GI) de forma randomizada por sequência simples, gerada por computador no site "www.randomization.com" por um pesquisador não envolvido nas análises dos dados.

O GI foi acompanhado e orientado por enfermeiros de forma presencial e telefônica. A consulta de Enfermagem presencial ocorreu antes da alta hospitalar, até 7 dias e em 30 dias após alta. Nestas consultas, foi realizado o exame físico cardiorrespiratório com aplicação do Escore Clínico de Congestão, seguido da aplicação de alguns questionários (Autocuidado, Adesão terapêutica, Conhecimento da doença e QV) e intervenção educativa subsidiada por cartilha educativa construída pelos pesquisadores deste estudo. Quanto aos questionários, foram considerados o questionário de autocuidado (sendo adequado quando pontuação $\geq 70$ ) e o de QV (classificada como boa quando escores até 26 pontos, moderada quando 26 a 45 pontos e ruim acima de 45 pontos) (Riegel et.al., 2009; Ávila, 2012; Behlouli et.al., 2009; Rector \& Conh, 1992; Carvalho et. al., 2009).

A intervenção educativa se deu com entrega de cartilha educativa e leitura em pares (Pesquisador interventor e paciente), sendo explicada de forma detalhada todas as estruturas textuais e imagens impressas na cartilha. Os conteúdos foram abordados de forma cronológica intercalada com imagens didáticas e textos informativos sobre atividade física, hábito alimentar, controle do peso, automedicação, compreensão do efeito terapêutico medicamentoso, sintomas clínicos de descompensação da IC e busca ao atendimento de saúde. Após o preenchimento dos instrumentos e intervenção educativa, foi entregue uma ficha para controle do peso e de registro de internação hospitalar para preenchimento exclusivo do paciente/cuidador. Por fim, a avaliação presencial se encerrava e o paciente era informado acerca da frequência das ligações que viriam ocorrer no $15^{\circ}$ e $45^{\circ}$ dias após alta.

As ligações telefônicas ocorreram com intuito educativo. O pesquisador interventor obedeceu ao protocolo das ligações, cujo conteúdo impresso nas cartilhas educativas foi discutido em forma de diálogo, a fim de reforçar as orientações da primeira consulta. Concomitante à intervenção por via telefônica, foi preenchido uma ficha de avaliação e acompanhamento padronizado para identificação das mudanças comportamentais no cuidado e manejo da doença.

Os participantes foram reavaliados ao final com 60 dias após alta hospitalar pelo enfermeiro-interventor que não tinha conhecimento a qual grupo pertencia o paciente. Nesta reavaliação, foram aplicados os mesmos critérios supracitados (exame físico, escore clínico de congestão, aplicação dos questionários) e realizada nova orientação educativa subsidiada pela cartilha educativa e análise dos desfechos alcançados. O fluxograma da intervenção do GI é apresentado na Figura 1. 
Figura1. Fluxograma das intervenções realizadas ao Grupo Intervenção.

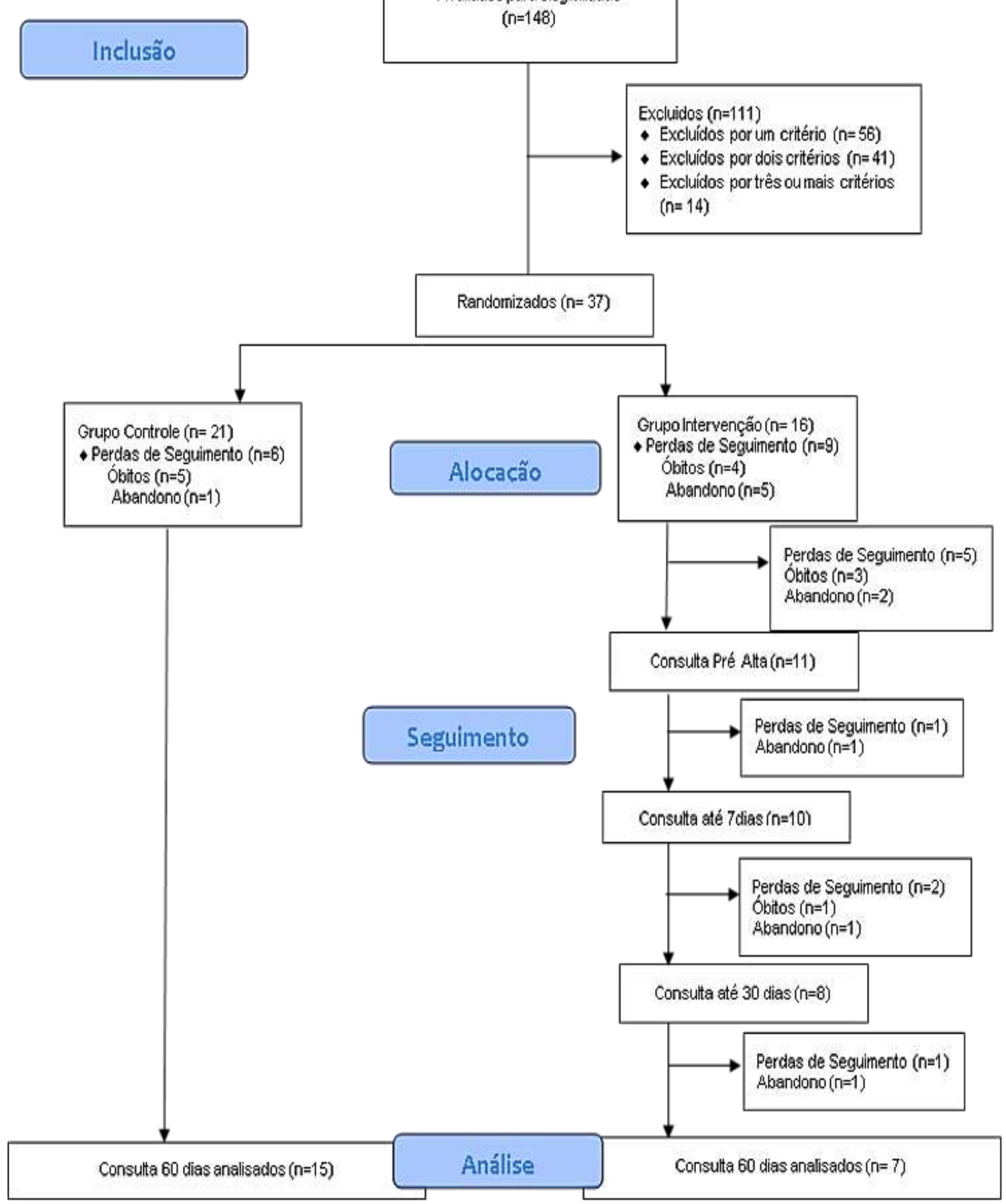

Fonte: Dados da pesquisa (2021).

Quanto ao grupo controle, os participantes não receberam orientações educativas, apenas foram informados sobre a necessidade de registro do número e duração de reinternações após entrada na pesquisa, bem como sobre a reavaliação presencial com 60 dias após alta hospitalar. A avaliação final com 60 dias ocorreu de forma idêntica à do GI. 
Variáveis categóricas foram dispostas em números absolutos (n) e frequência relativa (\%). Variáveis contínuas foram descritos em medidas de tendência central. Para avaliar a associação, foi realizado teste Qui-quadrado de Pearson e a razão de verossimilhança. Os dados foram analisados por meio da ferramenta SPSS - Statistical Package for Social Sciences 21.0.

\section{Resultados e Discussão}

Os resultados apresentados a seguir correspondem aos pacientes avaliados durante o período de dezembro de 2018 a março de 2020. Neste período, 148 pacientes foram admitidos por descompensação clínica da IC, sendo elegíveis à coleta. Dentre os 148, 111 foram excluídos conforme critérios pré-estabelecidos: 56 (38\%) excluídos por somente um critério, 41 (28\%) por dois critérios e 14 (10\%) por três ou mais critérios. Dessa maneira, 37 (24\%) foram incluídos de forma randomizada: 21 (57\%) no GC e 16 (43\%) GI. Aos 60 dias pós alta, foram avaliados 15 pacientes no GC e 7 no GI. A Figura 2 apresenta o fluxograma da inclusão, alocação, seguimento e análise para o ECR.

Figura 2. Fluxograma CONSORT.

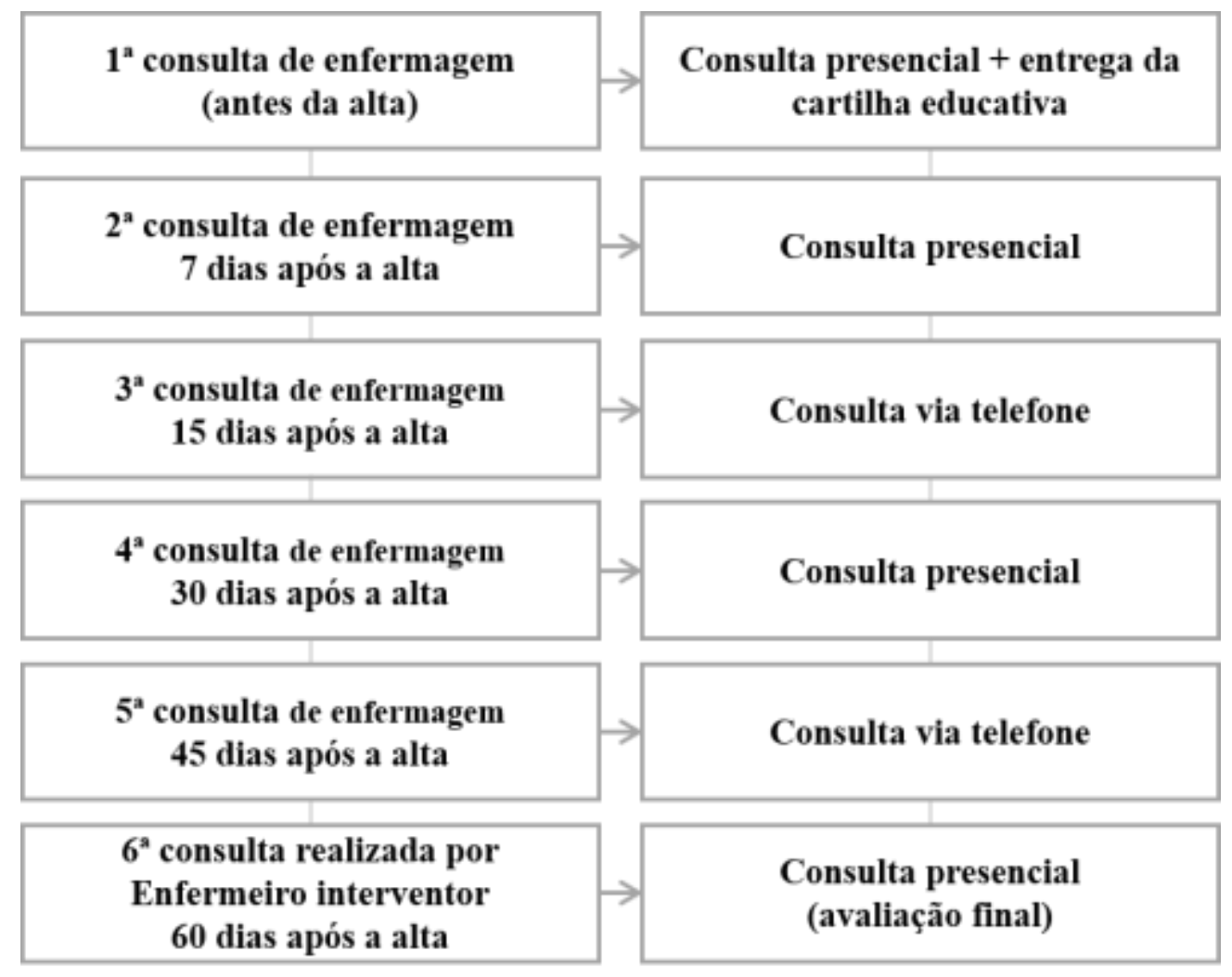

Fonte: CONSORT (2010).

No GI em comparação GC, predominou-se o sexo feminino (56\% vs 38\%), com média de idade de $65 \pm 14$ vs $63 \pm 14$ anos, de raça parda (44\% vs 43), escolaridade de 1 a 7 anos (44\% vs 47\%), ex tabagistas (56\% vs $72 \%$ ), ex etilistas (44\% vs $28 \%$ ), não praticantes de atividades físicas (75\% vs $90 \%)$. No que se refere às características clínicas do GI em comparação ao GC, foi identificada predominância do tempo de até 1 ano de diagnóstico da IC (38\% vs 14\%), etiologia isquêmica (44\% vs

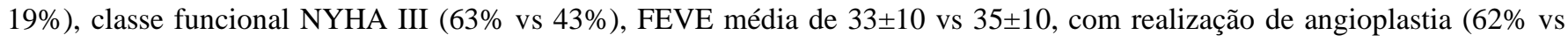
$38 \%$ ), principais comorbidades a Hipertensão Arterial Sistêmica (81\% vs 62\%) e Diabetes Mellitus (37\% vs 24) e média de $21 \pm 4$ vs $21 \pm 4$ no nível de cognição avaliado pelo MEEM. Os demais dados referentes às características clínicas, demográficas e o estilo de vida destes pacientes estão dispostos na Tabela 1. 
Research, Society and Development, v. 10, n. 16, e276101623445, 2021

(CC BY 4.0) | ISSN 2525-3409 | DOI: http://dx.doi.org/10.33448/rsd-v10i16.23445

Tabela 1. Caracterização clínica e demográfica dos pacientes de acordo com os grupos.

\begin{tabular}{|c|c|c|c|}
\hline VARIÁVEIS & $\begin{array}{c}\text { GC } \\
(n=21)\end{array}$ & $\begin{array}{c}\text { GI } \\
(n=16)\end{array}$ & p valor \\
\hline \multicolumn{4}{|l|}{ Sexo } \\
\hline Masculino, n (\%) & $13(62)$ & $7(44)$ & \multirow{2}{*}{0,331} \\
\hline Feminino, $\mathrm{n}(\%)$ & $8(38)$ & $9(56)$ & \\
\hline Idade, média anos \pm Desvio Padrão & $63 \pm 14$ & $65 \pm 14$ & 0,904 \\
\hline \multicolumn{4}{|l|}{ Raça } \\
\hline Pardo, n (\%) & $9(43)$ & $7(44)$ & \multirow{3}{*}{0,525} \\
\hline Negro, n (\%) & $7(33)$ & $3(19)$ & \\
\hline Branco, n (\%) & $5(24)$ & $6(37)$ & \\
\hline \multicolumn{4}{|l|}{ Escolaridade } \\
\hline Analfabeto, n (\%) & $6(29)$ & $6(37)$ & \multirow{3}{*}{0,835} \\
\hline 1 a 7 anos, n (\%) & $10(47)$ & $7(44)$ & \\
\hline$\geq 8$ anos, $\mathrm{n}(\%)$ & $5(24)$ & $3(19)$ & \\
\hline \multicolumn{4}{|l|}{ Mora com } \\
\hline Sozinho, n (\%) & $5(24)$ & $3(19)$ & \multirow{2}{*}{1} \\
\hline Família, n (\%) & $16(76)$ & $13(81)$ & \\
\hline \multicolumn{4}{|l|}{ Renda familiar } \\
\hline Até 1 salário mínimo, n (\%) & $9(43)$ & $11(69)$ & \multirow[t]{2}{*}{0,406} \\
\hline 1 a 2 salários mínimos, n (\%) & $8(38)$ & $4(25)$ & \\
\hline \multicolumn{4}{|l|}{ Tabagismo } \\
\hline Ex-tabagista, n (\%) & $15(72)$ & $9(56)$ & \multirow{2}{*}{0,489} \\
\hline Não, $\mathrm{n}(\%)$ & $6(28)$ & $7(44)$ & \\
\hline \multicolumn{4}{|l|}{ Etilismo } \\
\hline Sim, n (\%) & $5(17)$ & $3(19)$ & \multirow[t]{3}{*}{0,914} \\
\hline Não, n (\%) & $8(28)$ & $6(37)$ & \\
\hline \multirow[t]{3}{*}{ Ex etilista, $\mathrm{n}(\%)$} & $8(28)$ & $7(44)$ & \\
\hline & GC & GI & \multirow[b]{2}{*}{ p valor } \\
\hline & $(\mathrm{n}=21)$ & $(\mathrm{n}=16)$ & \\
\hline Já tentou parar de beber, n (\%) & $1(3)$ & $0(0)$ & 0,858 \\
\hline Já recebeu aconselhamento para parar de beber, n (\%) & $1(3)$ & $0(0)$ & 1 \\
\hline Atividade física, n (\%) & $2(10)$ & $4(25)$ & 0,371 \\
\hline \multicolumn{4}{|l|}{ Tempo de IC } \\
\hline Até 1 ano, n (\%) & $3(14 \%)$ & $6(38 \%)$ & \multirow{4}{*}{0,352} \\
\hline 1 a 5 anos, n (\%) & $5(24 \%)$ & $4(25 \%)$ & \\
\hline$>5$ anos, $\mathrm{n}(\%)$ & $10(48 \%)$ & $4(25 \%)$ & \\
\hline Não sabe, n (\%) & $3(14 \%)$ & $2(12 \%)$ & \\
\hline \multicolumn{4}{|l|}{ Etiologia } \\
\hline Isquêmica, n (\%) & $4(19 \%)$ & $7(44 \%)$ & \\
\hline Hipertensiva, n (\%) & $7(33 \%)$ & $5(31 \%)$ & 0,386 \\
\hline
\end{tabular}


Research, Society and Development, v. 10, n. 16, e276101623445, 2021

(CC BY 4.0) | ISSN 2525-3409 | DOI: http://dx.doi.org/10.33448/rsd-v10i16.23445

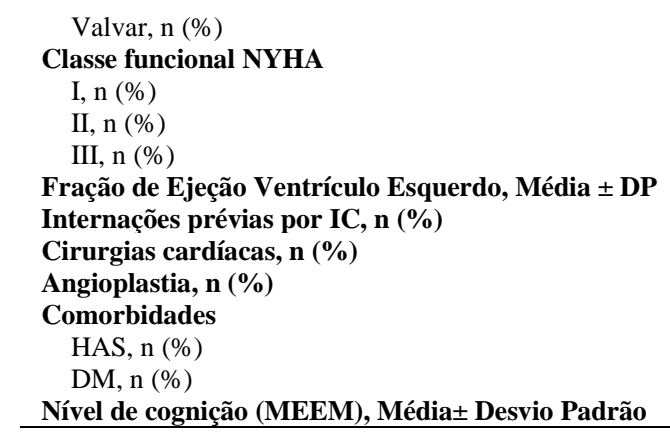

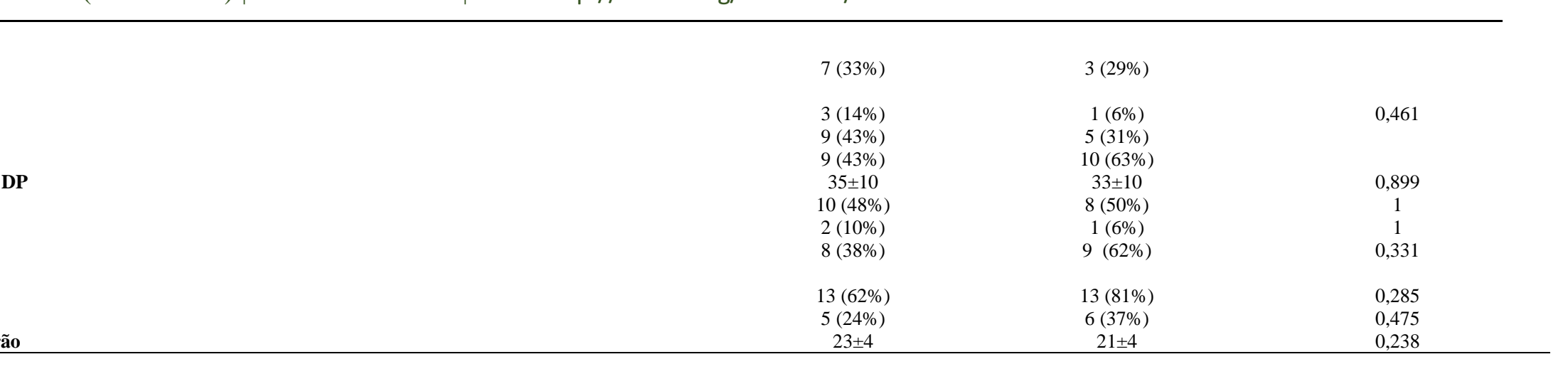

Fonte: Dados da pesquisa (2021).

Quanto à avaliação do autocuidado, o instrumento é dividido em três seções, sendo A sobre manutenção do autocuidado, B sobre manejo e C sobre confiança na prática do autocuidado. Neste sentido, na Seção A destacaram-se melhores práticas de autocuidado no GI em comparação ao GC nos itens relacionados aos hábitos de: se pesar frequentemente (43\% vs $20 \%$ ), sempre ou diariamente observam se os tornozelos estão inchados (GI 71\% vs GC 47\%), tentam evitar ficar doente (GI 57\% vs GC27\%), realizam alguma atividade física (GI 57\% vs GC 75\%), assiduidade nas consultas (GI 86\% vs GC 67\%), possui dieta com pouco sal (GI 100\% vs GC 40\%), solicitam alimentos com pouco sal quando come fora ou visita alguém (GI 86\% vs GC 27\%), usam algum sistema para ajudar a lembrar dos medicamentos (GI 71\% vs GC 60\%). Na seção B, destacaram-se melhores práticas de autocuidado no GI em comparação ao GC nos itens relacionados a redução do consumo de sal (GI 100\% vs GC 53\%), redução da ingesta hídrica (GI 86\% vs GC 27\%) e os que contataram seu médico ou enfermeiro para orientações (GI 100\% vs GC 60\%). Na seção C, destacaram-se melhores práticas de autocuidado no GI em comparação ao GC nos itens relacionados estarem livres dos sintomas da IC (GI $57 \%$ vs GC $34 \%$ ), seguem o tratamento recomendado (GI $86 \%$ vs GC 40\%), reconhecem as alterações de saúde (GI 86\% vs GC 26\%), fazem algo que possam aliviar os sintomas (GI $43 \%$ vs GC 33\%) e avaliam se o medicamento funciona (GI 57\% vs GC 20\%). Demais itens estão apresentados na Tabela 2. 
Research, Society and Development, v. 10, n. 16, e276101623445, 2021

(CC BY 4.0) | ISSN 2525-3409 | DOI: http://dx.doi.org/10.33448/rsd-v10i16.23445

Tabela 2: Autocuidado dos pacientes de acordo com os grupos.

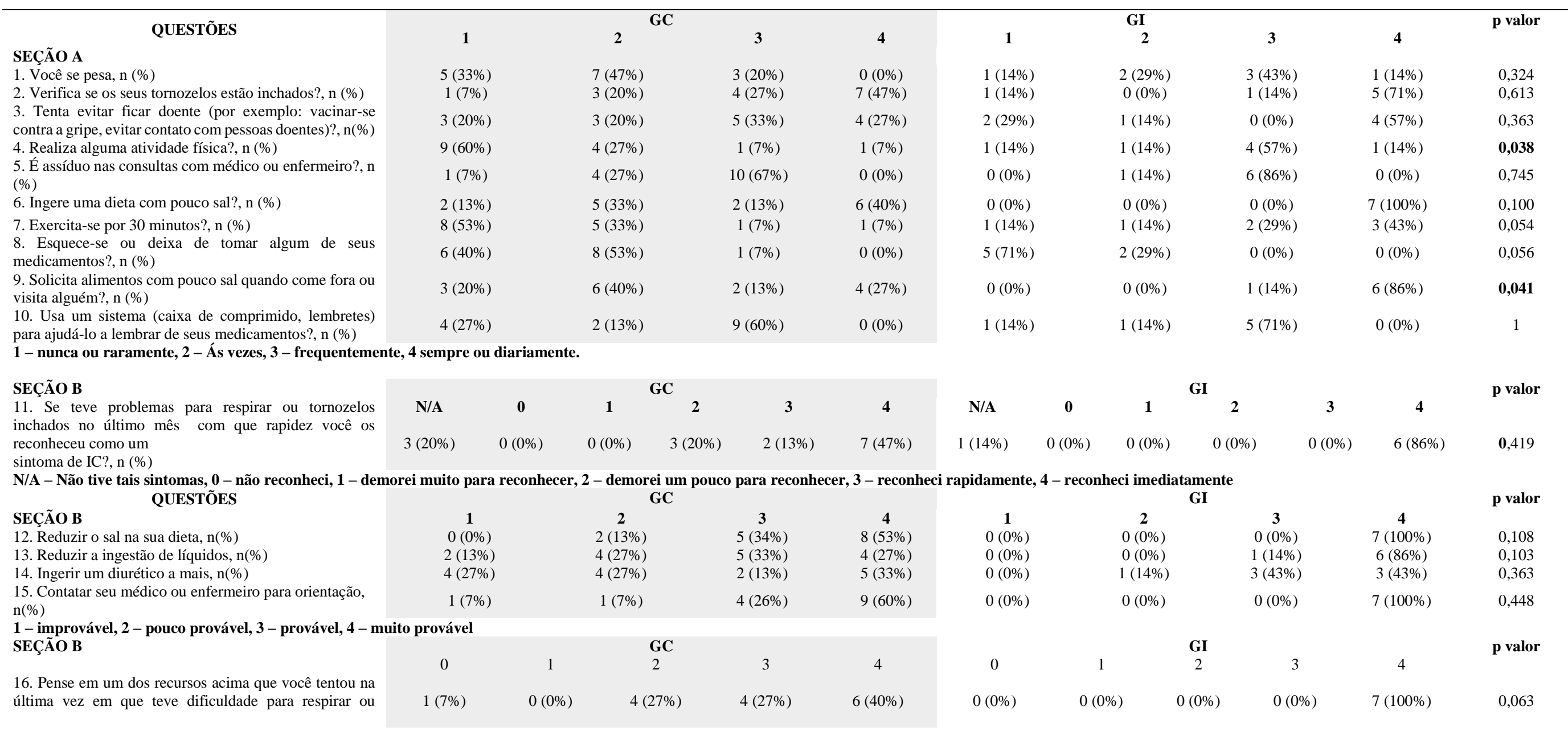


Research, Society and Development, v. 10, n. 16, e276101623445, 2021

(CC BY 4.0) | ISSN 2525-3409 | DOI: http://dx.doi.org/10.33448/rsd-v10i16.23445

tornozelos inchados. Você tem certeza de que este recurso o ajudou?

0 - eu não tentei nada, 1 - não tenho certeza, 2 - tenho certeza, 3 - tenho pouca certeza, 4 - tenho absoluta certeza Seção C

17. Estar livre dos sintomas de insuficiência cardíaca? 18. Seguir o tratamento recomendado?

19. Avaliar a importância de seus sintomas?

20. Reconhecer alterações na saúde, caso elas ocorram?

21. Fazer algo que possa avaliar seus sintomas?

22. Avaliar se um medicamento funciona?

$\mathbf{1}$
$2(13 \%)$
$0(0 \%)$
$0(0 \%)$
$0(0 \%)$
$1(7 \%)$
$1(7 \%)$

$\underset{2}{\mathbf{G C}}$
$6(40 \%)$
$2(13 \%)$
$4(27 \%)$
$4(27 \%)$
$4(27 \%)$
$7(47 \%)$

$\begin{array}{cc}\mathbf{3} & \mathbf{4} \\ 5(33 \%) & 2(13 \%) \\ 7(47 \%) & 6(40 \%) \\ 8(53 \%) & 3(20 \%) \\ 7(47 \%) & 4(27 \%) \\ 5(33 \%) & 5(33 \%) \\ 4(27 \%) & 3(20 \%)\end{array}$

$\mathbf{1}$
$0(0 \%)$
$0(0 \%)$
$0(0 \%)$
$0(0 \%)$
$0(0 \%)$
$0(0 \%)$

$\underset{2}{ }$
$0(0 \%)$
$1(14 \%)$
$0(0 \%)$
$0(0 \%)$
$1(14 \%)$
$1(14 \%)$

GI

\begin{tabular}{ccc}
$\mathbf{3}$ & $\mathbf{4}$ & p valor \\
$4(57 \%)$ & $3(43 \%)$ & 0,113 \\
$0(0 \%)$ & $6(86 \%)$ & 0,079 \\
$3(43 \%)$ & $4(57 \%)$ & 0,188 \\
$1(14 \%)$ & $6(86 \%)$ & 0,044 \\
$3(43 \%)$ & $3(43 \%)$ & 1 \\
$2(29 \%)$ & $4(57 \%)$ & 0,302 \\
\hline
\end{tabular}

1 - não confiante, 2 - um pouco confiante, 3 - muito confiante, 4 - extremamente confiante

Fonte: Dados da pesquisa (2021).

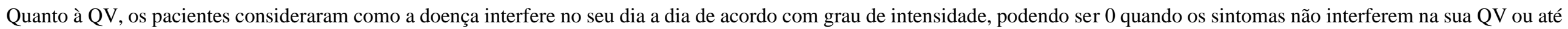

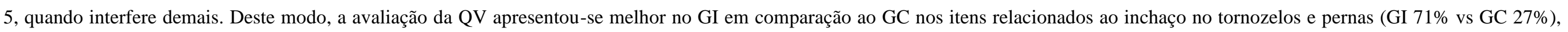

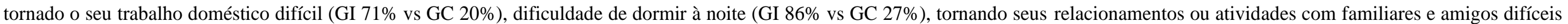

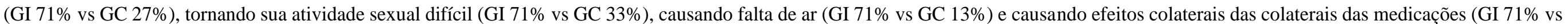
GC 80\%). Demais informações estão descritas na Tabela 3. 
Research, Society and Development, v. 10, n. 16, e276101623445, 2021

Tabela 3. Qualidade de vida dos pacientes portadores de IC de acordo com os grupos.

\section{QUESTÕES}

1.Causou inchaço em seus tornozelos e pernas, $\mathrm{n}(\%)$

2. Obrigando você a sentar ou deitar para descansar durante o dia, $\mathrm{n}(\%)$

3. Tornando sua caminhada e subida de escadas difícil,

$\mathrm{n}(\%)$

4. Tornando seu trabalho doméstico difícil, n(\%)

5. Tornando suas saídas de casa difíceis, n(\%)

6. Tornando difícil dormir bem à noite, $\mathrm{n}(\%)$

7. Tornando seus relacionamentos ou atividades com familiares e amigos difíceis, $\mathrm{n}(\%)$

8. Tornando seu trabalho para ganhar a vida difícil, $\mathrm{n}(\%)$

9. Tornando seus passatempos, esportes e diversão difíceis, $\mathrm{n}(\%)$

10. Tornando sua atividade sexual difícil, $\mathrm{n}(\%)$

11. Fazendo você comer menos as comidas que você gosta $\mathrm{n}(\%)$

12. Causando falta de ar, $\mathrm{n}(\%)$

13. Deixando você cansado, fadigado ou com pouca energia, $\mathrm{n}(\%)$

14. Fazendo você gastar dinheiro com cuidados médicos, $\mathrm{n}(\%)$

15. Causando a você efeitos colaterais das medicações,

$\mathrm{n}(\%)$

16. Fazendo você sentir-se um peso para familiares e

amigos, $\mathrm{n}(\%)$

17. Fazendo você sentir uma falta de auto controle na sua vida, $\mathrm{n}(\%)$

18. Fazendo você se preocupar, n(\%)

\begin{tabular}{|c|c|c|c|c|c|c|}
\hline \multicolumn{7}{|c|}{ GC } \\
\hline 0 & 1 & 2 & 3 & 4 & 5 & 0 \\
\hline 4 & 3 & 5 & 1 & 1 & 1 & 5 \\
\hline$(27 \%)$ & $(20 \%)$ & $(33 \%)$ & $(7 \%)$ & $(7 \%)$ & $(7 \%)$ & $(71 \%)$ \\
\hline 2 & 2 & 1 & 3 & 4 & 3 & 3 \\
\hline$(13 \%)$ & $(13 \%)$ & $(7 \%)$ & $(20 \%)$ & $(27 \%)$ & $(20 \%)$ & $(43 \%)$ \\
\hline 2 & 2 & 1 & 3 & 3 & 4 & 2 \\
\hline$(13 \%)$ & $(13 \%)$ & $(7 \%)$ & $(20 \%)$ & $(20 \%)$ & $(27 \%)$ & $(29 \%)$ \\
\hline 3 & 1 & 4 & 3 & 2 & 2 & 5 \\
\hline$(20 \%)$ & $(7 \%)$ & $(27 \%)$ & $(20 \%)$ & $(13 \%)$ & $(13 \%)$ & $(71 \%)$ \\
\hline 2 & 3 & 2 & 5 & 2 & 1 & 4 \\
\hline$(13 \%)$ & $(20 \%)$ & $(13 \%)$ & $(33 \%)$ & $(13 \%)$ & $(7 \%)$ & $(57 \%)$ \\
\hline 4 & 3 & 3 & 3 & 1 & 1 & 6 \\
\hline$(27 \%)$ & $(20 \%)$ & $(20 \%)$ & $(20 \%)$ & $(7 \%)$ & $(7 \%)$ & $(86 \%)$ \\
\hline 4 & 3 & 7 & 1 & 0 & 0 & 5 \\
\hline$(27 \%)$ & $(20 \%)$ & $(46 \%)$ & $(7 \%)$ & $(0 \%)$ & $(0 \%)$ & $(71 \%)$ \\
\hline 5 & 1 & 2 & 1 & 2 & 4 & 4 \\
\hline$(33 \%)$ & $(7 \%)$ & $(13 \%)$ & $(7 \%)$ & $(13 \%)$ & $(27 \%)$ & $(57 \%)$ \\
\hline 2 & 2 & 5 & 2 & 0 & 4 & 4 \\
\hline$(13 \%)$ & $(13 \%)$ & $(33 \%)$ & $(13 \%)$ & $(0 \%)$ & $(27 \%)$ & $(57 \%)$ \\
\hline 5 & 0 & 3 & 0 & 1 & 6 & 5 \\
\hline$(33 \%)$ & $(0 \%)$ & $(20 \%)$ & $(0 \%)$ & $(7 \%)$ & $(40 \%)$ & $(71 \%)$ \\
\hline 4 & 1 & 3 & 2 & 3 & 2 & 2 \\
\hline$(27 \%)$ & (7\%) & $(20 \%)$ & $(13 \%)$ & $(20 \%)$ & $(13 \%)$ & $(29 \%)$ \\
\hline 2 & 3 & 2 & 3 & 1 & 4 & 5 \\
\hline$(13 \%)$ & $(20 \%)$ & $(13 \%)$ & $(20 \%)$ & $(7 \%)$ & $(27 \%)$ & $(71 \%)$ \\
\hline 1 & 2 & 1 & 4 & 3 & 4 & 4 \\
\hline$(7 \%)$ & $(13 \%)$ & $(7 \%)$ & $(27 \%)$ & $(20 \%)$ & $(27 \%)$ & $(57 \%)$ \\
\hline 2 & 0 & 0 & 3 & 1 & 9 & 0 \\
\hline$(13 \%)$ & $(0 \%)$ & $(0 \%)$ & $(20 \%)$ & $(7 \%)$ & $(60 \%)$ & $(0 \%)$ \\
\hline 12 & 0 & 1 & 1 & 1 & 0 & 5 \\
\hline (80) & $(0 \%)$ & $(7 \%)$ & $(7 \%)$ & $(6 \%)$ & $(0 \%)$ & $(71 \%)$ \\
\hline 5 & 2 & 4 & 1 & 1 & 2 & 4 \\
\hline$(33 \%)$ & $(13 \%)$ & $(27 \%)$ & $(7 \%)$ & $(7 \%)$ & $(13 \%)$ & $(57 \%)$ \\
\hline 3 & 0 & 5 & 4 & 0 & 3 & 3 \\
\hline$(20 \%)$ & $(0 \%)$ & $(33 \%)$ & $(27 \%)$ & $(0 \%)$ & $(20 \%)$ & $(43 \%)$ \\
\hline 2 & 0 & 4 & 6 & 0 & 3 & 1 \\
\hline
\end{tabular}

GI

$\begin{array}{ccccc}\mathbf{1} & \mathbf{2} & \mathbf{3} & \mathbf{4} & \mathbf{5} \\ 4 & 0 & 0 & 0 & 0 \\ (29 \%) & (0 \%) & (0 \%) & (0 \%) & (0 \%) \\ 2 & 0 & 1 & 0 & 1 \\ (29 \%) & (0 \%) & (14 \%) & (0 \%) & (14 \%) \\ 4 & 0 & 0 & 1 & 0 \\ (57 \%) & (0 \%) & (0 \%) & (14 \%) & (0 \%) \\ 0 & 0 & 1 & 1 & 0 \\ (0 \%) & (0 \%) & (14 \%) & (14 \%) & (0 \%) \\ 2 & 0 & 0 & 1 & 0 \\ (29 \%) & (0 \%) & (0 \%) & (14 \%) & (0 \%) \\ 1 & 0 & 0 & 0 & 0 \\ (14 \%) & (0 \%) & (0 \%) & (0 \%) & (0 \%) \\ 2 & 0 & 0 & 0 & 0 \\ (29 \%) & (0 \%) & (0 \%) & (0 \%) & (0 \%) \\ 0 & 1 & 0 & 0 & 2 \\ (0 \%) & (14 \%) & (0 \%) & (0 \%) & (29 \%) \\ 1 & 2 & 0 & 0 & 0 \\ (14 \%) & (29 \%) & (0 \%) & (0 \%) & (0 \%) \\ 1 & 0 & 1 & 0 & 0 \\ (14 \%) & (0 \%) & (14 \%) & (0 \%) & (0 \%) \\ 0 & 1 & 0 & 0 & 4 \\ (0 \%) & (14 \%) & (0 \%) & (0 \%) & (57 \%) \\ 2 & 0 & 0 & 0 & 0 \\ (29 \%) & (0 \%) & (0 \%) & (0 \%) & (0 \%) \\ 2 & 0 & 1 & 0 & 0 \\ (29 \%) & (0 \%) & (14 \%) & (0 \%) & (0 \%) \\ 1 & 1 & 0 & 0 & 5 \\ (14 \%) & (14 \%) & (0 \%) & (0 \%) & (71 \%) \\ 2 & 0 & 0 & 0 & 0 \\ (29 \%) & (0 \%) & (0 \%) & (0 \%) & (0 \%) \\ 2 & 0 & 0 & 0 & 1 \\ (29 \%) & (0 \%) & (0 \%) & (0 \%) & (14 \%) \\ 2 & 0 & 0 & 1 & 1 \\ (29 \%) & (0 \%) & (0 \%) & (14 \%) & (14 \%) \\ 2 & 0 & 1 & 1 & 2 \\ & & & & \\ 2 & & 0\end{array}$

P valor

0,331

0,573

0,203

0,286

0,230

0,250

0,091

0,952

0,232

0,028

0,382

0,089

0,069

0,212

0,304

0,652

0,027

0,089 
Research, Society and Development, v. 10, n. 16, e276101623445, 2021 (CC BY 4.0) | ISSN 2525-3409 | DOI: http://dx.doi.org/10.33448/rsd-v10i16.23445

\begin{tabular}{|c|c|c|c|c|c|c|c|c|c|c|c|c|c|}
\hline & $(13 \%)$ & $(0 \%)$ & $(27 \%)$ & $(40 \%)$ & $(0 \%)$ & $(20 \%)$ & $(14 \%)$ & $(29 \%)$ & $(0 \%)$ & $(14 \%)$ & $(14 \%)$ & $(29 \%)$ & \\
\hline $\begin{array}{l}\text { 19. Tornando difícil você concentrar-se ou lembrar-se das } \\
\text { coisas, } \mathrm{n}(\%)\end{array}$ & $\begin{array}{c}4 \\
(27 \%)\end{array}$ & $\begin{array}{c}2 \\
(13 \%)\end{array}$ & $\begin{array}{c}1 \\
(7 \%)\end{array}$ & $\begin{array}{c}5 \\
(33 \%)\end{array}$ & $\begin{array}{c}2 \\
(13 \%)\end{array}$ & $\begin{array}{c}1 \\
(7 \%)\end{array}$ & $\begin{array}{c}3 \\
(43 \%)\end{array}$ & $\begin{array}{c}2 \\
(29 \%)\end{array}$ & $\begin{array}{c}0 \\
(0 \%)\end{array}$ & $\begin{array}{c}0 \\
(0 \%)\end{array}$ & $\begin{array}{c}0 \\
(0 \%)\end{array}$ & $\begin{array}{c}2 \\
(29 \%)\end{array}$ & 0,269 \\
\hline OUESTÕES & \multicolumn{6}{|c|}{ GC } & \multicolumn{5}{|c|}{ GI } & \multirow{2}{*}{\multicolumn{2}{|c|}{ P Valor }} \\
\hline QUES TUES & $\mathbf{0}$ & 1 & 2 & 3 & 4 & 5 & $\mathbf{0}$ & 1 & 2 & 3 & 4 & & \\
\hline 20. Fazendo você sentir-se deprimido, n(\%) & $\begin{array}{c}6 \\
(40 \%)\end{array}$ & $\begin{array}{c}2 \\
(13 \%)\end{array}$ & $\begin{array}{c}2 \\
(13 \%)\end{array}$ & $\begin{array}{c}0 \\
(0 \%)\end{array}$ & $\begin{array}{c}4 \\
(27 \%)\end{array}$ & $\begin{array}{c}1 \\
(7 \%)\end{array}$ & $\begin{array}{c}3 \\
(43 \%)\end{array}$ & $\begin{array}{c}1 \\
(14 \%)\end{array}$ & $\begin{array}{c}2 \\
(29 \%)\end{array}$ & $\begin{array}{c}0 \\
(0 \%)\end{array}$ & $\begin{array}{c}0 \\
(0 \%)\end{array}$ & $\begin{array}{c}1 \\
(14 \%)\end{array}$ & 0,653 \\
\hline
\end{tabular}

Não, 1-Muito Pouco, 2-Se maior que 1, 3-Se maior que 2, 4-Se maior que 3, 5-Demais.

Fonte: Dados da pesquisa (2021). 
A Tabela 4 descreve os escores de congestão, autocuidado e QV destes pacientes aos 60 dias pós alta. Quanto à congestão clínica, os pacientes do GI tiveram melhor apresentação clínica em comparação ao GC, evidenciado por totalidade de indivíduos não congestos $(100 \%, \mathrm{n}=7)$ em comparação aos $53 \%(\mathrm{n}=8)$ no GC. No que se refere à QV, o GC apresentou maiores escores na avaliação ruim da QV $(60 \%, n=9)$. No GI houve distribuição equilibrada entre as avaliações, não havendo significância estatística na comparação entre os grupos $(\mathrm{p}=0,36)$. Os grupos GC e GI expressaram resultados opostos e estatisticamente significativos $(\mathrm{p}=0,02)$ quanto ao autocuidado, predominando um autocuidado adequado no GI 7 (100\%) enquanto no GC, autocuidado inadequado $(53 \%, \mathrm{n}=11)$.

Tabela 4.

\begin{tabular}{|c|c|c|c|}
\hline ESCORES & $\begin{array}{c}\text { GC } \\
(n=15)\end{array}$ & $\begin{array}{c}\text { GI } \\
(\mathbf{n}=7)\end{array}$ & $\begin{array}{l}\text { p valor } \\
(<0,05)\end{array}$ \\
\hline \multicolumn{4}{|l|}{ Congestão clínica } \\
\hline Congesto & $7(47 \%)$ & $0(0 \%)$ & \multirow{2}{*}{0,05} \\
\hline Não congesto & $8(53 \%)$ & $7(100 \%)$ & \\
\hline \multicolumn{4}{|l|}{ Qualidade de vida } \\
\hline Boa, n (\%) & $3(20 \%)$ & $3(44 \%)$ & \multirow{3}{*}{0,36} \\
\hline Moderada, n (\%) & $3(20 \%)$ & $2(28 \%)$ & \\
\hline Ruim, n (\%) & $9(60 \%)$ & $2(28 \%)$ & \\
\hline \multicolumn{4}{|l|}{ Autocuidado } \\
\hline Adequado, n (\%) & $7(47 \%)$ & $7(100 \%)$ & \multirow{2}{*}{$\mathbf{0 , 0 2}$} \\
\hline Não adequado, n (\%) & $11(53 \%)$ & $0(0 \%)$ & \\
\hline
\end{tabular}

Fonte: Dados da pesquisa (2021).

Neste estudo, avaliou-se a eficácia de uma intervenção educativa de Enfermagem no pós alta hospitalar de pacientes com IC. Ao analisar as amostras dos GI e GC, foi possível identificar o perfil sociodemográfico e clínico dos pacientes. Houve predominância da IC em indivíduos do sexo masculino (54\%, n=20), idosos (GI 65 vs GC 63) e com menos de 8 anos de escolaridade (GI 81\% vs GC 76\%). Contudo, não foi evidenciada significância estatística entre os grupos.

Indivíduos com baixa escolaridade, principalmente do sexo masculino, são mais suscetíveis à exposição aos fatores de riscos das doenças cardiovasculares (DCV’s), em decorrência das peculiaridades do seu perfil demográfico e epidemiológico (Simão et. al., 2013). Contudo, por apresentarem uma expectativa de vida maior à masculina, as mulheres com IC tendem a ser mais velhas e apresentam associação com outras comorbidades com hipertensão (Mantovani et. al., 2015; Oliveira et. al., 2017).

O nível educacional reflete diretamente na saúde do indivíduo; dessa forma, as práticas de educação em saúde no âmbito da atenção primária a saúde é importante para o tratamento de pacientes diagnosticados com IC. Uma coorte retrospectiva apontou uma correlação negativa mostrando que quanto menor educação em saúde, maiores taxas de mortalidade em portadores de IC (Qavan et. al., 2017). Portanto, é importante direcionar a atenção à saúde para as populações com baixo grau de escolaridade, para que possam ter entendimento sobre suas condições de saúde por meio de políticas públicas.

Em relação à etnia dos portadores de IC, houve predomínio de participantes autodeclarados pardos em ambos os grupos (GI 44\% e GC 43\%). Os dados epidemiológicos indicam que pacientes negros e brancos com IC tendem a ter mais fatores de riscos relacionados a DCV's, incluindo menor fração de ejeção e comorbidades, como hipertensão arterial sistólica e diabetes mellitus (Arruda et.al., 2017). Um estudo de coorte mostrou que pacientes da etnia negra têm maior taxa de readmissão comparado às pessoas brancas, independente de fatores socioeconômicos e características do hospital em que está internado (Srisuk et.al., 2016).

Fatores socioeconômicos também foram analisados neste estudo. Foi percebido que em ambos os grupos houve predomínio de pacientes com baixa renda, evidenciado pelo relato de 11 participantes do GI (69\%) e 9 do GC (43\%) receberem 
até um salário mínimo. Estudos mostram que a renda familiar é um dos principais indicadores que descreve a classe econômica e pacientes que possuem baixa renda tem maiores taxas de readmissões (Tsanani et. al., 2016; Hua et. al., 2017).

Outros estudos relatam que os indicadores socioeconômicos no tocante à renda são de extrema relevância, visto que melhor renda proporciona acessos aos bens e serviços e que a desigualdade de renda está intimamente associada às altas taxas de mortalidade (Mantovani et. al., 2015; Oliveira et. al., 2017). Diante disso, a procura dos serviços de saúde por motivos de prevenção é maior pela população de maior poder aquisitivo.

Quanto aos aspectos clínicos da IC deste estudo, foi possível perceber que a maioria dos pacientes do GI e GC foi classificado como NYHA III (63\%, n=10; 43\%, n=9, respectivamente). A classificação em classe funcional estabelecida pela NYHA permite identificar o quão o paciente está comprometido para realizar suas atividades de vida diária consequente às manifestações da doença. Deste modo, quanto mais alta a classificação, maior o grau de comprometimento. Um estudo de coorte prospectiva realizado na Índia que monitorou pacientes com IC com classe funcional III e IV durante um período de cinco anos constatou que 3 em cada 5 pacientes foram a óbito e a principal causa da mortalidade foi a descompensação do quadro clínico e falta de orientações destinadas ao manejo da doença (Harikrishnan et. al., 2020).

Nessa perspectiva, a interferência nas atividades de vida diária repercute na QV autorrelatada pelo paciente. Neste estudo, o GI apresentou melhores resultados no tocante à QV após as intervenções. Por outro lado, o GC não conseguiu apresentar melhora na QV. Um estudo demonstra que o acompanhamento dos portadores de IC em clinicas especializadas é fundamental para o aumento da QV dos mesmos (Bocchi et.al., 2008). Um estudo prospectivo, randomizado comparou pacientes portadores de IC submetidos a educação repetitiva e acompanhamento em clínicas especializadas com um GC. Destes, foi evidenciada maior QV nos pacientes que receberam a intervenção (Whitaker-brown et. al.,2017).

Paralelamente, sabe-se que a descompensação da doença pode estar atrelada à exacerbação dos sinais e sintomas como consequência do descumprimento da terapêutica proposta. Este estudo evidenciou que o autocuidado dos pacientes do GI apresentou melhora significativa quando comparados ao GC. Um estudo fez uma avaliação do autocuidado na IC como ferramenta fundamental na abordagem aos pacientes com tal síndrome, a fim de que enfermeiros possam contribuir efetivamente para o desenvolvimento das competências cognitivas e de instrumentos que facilitem a adoção de estratégias adaptativas, que contribuam para a manutenção do autocuidado, num processo de capacitação e autonomia. Ademais, as ações visam promover melhorias no autocuidado e consequentemente na qualidade de vida dos pacientes, tornando-os mais independentes nos comportamentos que levam à melhoria da sua saúde (Pereira, 2003).

Estudos revelam que o autocuidado de portadores de IC é inapropriado e que são necessárias estratégias de autogestão para melhorarias desses resultados (Qavan et.al., 2017; Arruda et.al., 2017). Nesta perspectiva, a educação em saúde, promovida por diferentes métodos, configura-se como uma importante estratégia de autogestão e que pode impactar positivamente no tratamento da IC e consequentemente, no melhor prognóstico do paciente.

Neste estudo, a educação em saúde foi realizada por enfermeiros através de VD e acompanhamento telefônico, cujas intervenções se mostraram eficazes na melhoria da QV de pacientes com IC. Além disso, dados do mesmo estudo revelam que as intervenções, quando realizadas pelos profissionais de enfermagem, por meio de VD, contribuem para o aumento da adesão ao tratamento e o autocuidado dessa população (Srisuk et.al., 2016).

Um Ensaio Clínico Randomizado (ECR) com portadores de IC que receberam intervenção domiciliar, encontros educativos, reforço com telemonitoramento e cartilha de orientações com seguimento de nove meses, evidenciou que o GI aumentou em $20 \%$ na escala de autocuidado, confirmando o efeito benéfico das abordagens (Tsanani et. al., 2016). O presente estudo corrobora com os resultados deste pois os pacientes tiveram aumento no escore do autocuidado de maneira significativa ao longo do tempo do estudo em cada consulta. 
Outro ECR utilizou como intervenção a monitorização telefônica quinzenal em 3 meses. Foi percebido que os escores de autocuidado foram maiores no GI do que no GC; $29 \%$ dos participantes receberam apenas 1 ou 2 contatos telefônicos em todo período de monitoramento (Srisuk et.al., 2016). Neste estudo, foram realizadas duas ligações telefônicas (a primeira no $15^{\circ}$ dia após a alta e a segunda no $45^{\circ}$ dia após a alta) e também foi evidenciada melhora no autocuidado do GI, confirmando assim o efeito positivo da abordagem realizada.

Estudos apontam a melhoria da QV como um dos objetivos mais importantes do tratamento na IC. Além disso, serve como uma medida de resultado fundamental em qualquer avaliação da eficácia do tratamento. Estratégias como o telemonitoramento e VD melhoraram consideravelmente a QV. Deste modo, novas formas de abordagens para tratamento da doença e consequente melhoria da QV são utilizadas na pratica clínica (Hua et. al., 2017; Dunbar et. al.,2015)

Um estudo de coorte, em que os pacientes foram acompanhados com a utilização do instrumento de QV, foi possível perceber melhora cerca de 30\% nos escores avaliados dos pacientes com IC, essa mudança pode estar intimamente relacionada ao aumento do autocuidado e melhora no quadro clinico (Tsanani et. al., 2016). Desse modo, o presente estudou indica que as intervenções lideradas por enfermeiros baseadas em orientações enfatizando o autocuidado e a disponibilização de folhetos educativos foram eficazes na melhoria dos componentes totais e no aspecto físico da QV.

Um ECR evidenciou que o uso de intervenção liderada por enfermeiras como educação em saúde e VD tem efeitos limitados sobre a QV em pacientes com IC no âmbito da atenção primária a saúde (Takeda, et al., 2019. O presente estudo se opõe desta informação pois os pacientes alocados no GI que recebeu as intervenções de enfermagem mostraram melhor QV. O grupo controle não apresentou uma boa QV.

Este estudo tem como principal limitação o tamanho da amostra, considerado pequeno para avaliação final. A redução da amostra esteve diretamente relacionada à perda por óbito durante o período de acompanhamento. Por tratar-se de um estudo de acompanhamento longo, entende-se que perdas por óbito ou abandono são passíveis de acontecer. Outro fato relacionado consiste na apresentação clínica da doença, visto que a maioria dos pacientes foi classificada como NYHA III. São necessários outros estudos com maior número de pacientes, porém os dados aqui apresentados puderam evidenciar benefícios quanto ao autocuidado e QV nos pacientes com IC após intervenção educativa.

\section{Considerações Finais}

A intervenção educativa realizada de forma presencial e telefônica por enfermeiros capacitados apresenta-se como uma importante ferramenta para melhora do autocuidado e QV. No tocante à QV, o GI apresentou em sua maioria boa qualidade de vida. Entretanto, não foi estatisticamente significante. Quanto ao autocuidado, este apresentou-se melhor no GI e estatisticamente significante.

Os dados deste estudo mostram que pacientes com IC tendem a possuir comportamento de autocuidado inadequado quando não acompanhados regularmente por profissionais de saúde, impactando em sua baixa QV frente às manifestações clínicas da doença. Deste modo, espera-se que os dados aqui apresentados sejam base para implantação de práticas educativas à este público, visando à capacitação dos pacientes para o devido reconhecimento e manejo dos sintomas. Ainda, sugere-se a realização de estudos voltados para essa temática no estado de Sergipe para construção de evidências cientificas e incorporação das intervenções de enfermagem. 


\section{Referências}

Amaral, D. R., Rossi, M. B., Lopes, C. T., \& Lopes, J. L. (2017) Intervenções não farmacológicas para melhor qualidade de vida na insuficiência cardíaca: revisão integrativa. Rev. Bras. Enferm. http://dx.doi.org/10.1590/0034-7167-2016-0112

Arruda, C. S., Perreira, J. M. V., Figueiredo, L. S., Scofano, B. S., Flores, P. V. P., \& Cavalcanti, A. C. D. (2017) Effect of an orientation group for patients with chronic heart failure: randomized controlled trial. Revista latino-americana de enfermagem. http://dx.doi.org/10.1590/1518-8345.2167.2982

Ávila, C. H. (2002). Adaptação transcultural e validação da Self Care of Heart Failure índex version 6.2 para uso no Brasil. Dissertação (Mestrado) - Curso de Enfermagem, Programa de Pós Graduação em Enfermagem, Universidade Federal de Porto Alegre, 2012.

Behlouli, H., Feldman, D. E., Ducharme, A., Frenette, M. Giannetti, Grondin, F., Michel, C., Sheppard, R., \& Pilote, L. (2009). Identifying relative cut-off scores with neurais networks for interpretation of the minnesota living with heart failure questionnaire. Annual International Conference Of The Ieee Engineering In Medicine And Biology Society. 10.1109/IEMBS.2009.5334659

Bertolucci, P. H. F., Brucki, S. M. D., Campacci, S. R., \& Julian, Y. (1994). O Mini-Exame do Estado Mental em uma população geral: impacto da escolaridade. Arquivos de Neuro-Psiquiatria. doi.org/10.1590/S0004-282X1994000100001.

Bocchi, E. A., Cruz, F., Guimarães, G., Moreira, L. F. P., Issa, V. S., Ferreira, S. M. A., Chizzola, P. R., Souza, G. E. C., Brandão, S., \& Bacal, F. (2008). A Long-term Prospective Randomized Controlled Study Using Repetitive Education at Six-Month Intervals and Monitoring for Adherence in Heart Failure Outpatients: The REMADHE Study. Circ Heart Fail. https://doi.org/10.1161/CIRCHEARTFAILURE.107.744870.

Boisvert, S., Belhumeur, A, B., Gonçalves, N., Doré, M., Francoeur, J., \& Gallani, M, C. (2015) Revisão integrativa sobre intervenções de enfermagem voltadas para a promoção do auto-cuidado entre pacientes portadores de insuficiência cardíaca. Rev. Latino-Am. Enfermagem. https:// 10.1590/0104-1169.0370.2612

Bozkurt, B., \& Khalaf, S. (2017) Heart failure in women. Methodist DeBakey cardiovascular journal. https// 10.14797/mdcj-13-4-216

Brasil, Ministério da Saúde. Secretaria Executiva. Datasus. Informações de Saúde. Sistema de Informações Hospitalares. Brasília (DF) 2021.

Brasil, Ministério da Saúde Secretaria Executiva. Datasus Informações de Saúde. Sistema de Informações sobre Mortalidade. Brasília (DF) 2021.

Brucki, S. M. D., Nitrini, R., Caramelli, P., Bertolucci, P. H. F., \& Okamoto, I. H. (2003). Sugestões para o uso do Mini-Exame do Estado Mental no Brasil. Arquivos de Neuro-Psiquiatria. doi.org/10.1590/S0004-282X2003000500014.

Carvalho, W. N., Gonçalves, K. C., Alves, G. S., Miranda, M. A. L., \& Moreira, M. C. V. (2020) Análise da qualidade de vida de pacientes com insuficiência cardíaca avançada candidatos ou não ao transplante cardíaco. REME - Rev Min Enferm. 10.5935/1415-2762.20200031

Creber, R. M., Patey, M., Lee, C. S., Kuan, A., Jurgens C., \& Riegel, B. (2016). Motivational interviewing to improve self-care for patients with chronic heart failure: MITI-HF randomized controlled trial. Patient Educ Couns. 99(2):256-64. https://doi.org/10.1016/j.pec.2015.08.031

Comitê Coordenador da Diretriz de Insuficiência Cardíaca. Diretriz Brasileira de Insuficiência Cardíaca Crônica e Aguda. Arq Bras Cardiol. 2018; 111(3):436539

Conceição, A. P., Santos, M. A., Santos, B., \& Cruz, D. A. L. M. (2015) Autocuidado de pacientes com insuficiência cardíaca. Rev. Latino-AM. Enfermagem. https:// 10.1590/0104-1169.0288.2591

Dainesi, S. M., \& Aligieri P. (2005) Como as recomendações "consort” podem assegurar a qualidade dos relados de estudos clínicos. Ver Assoc Med Bras. https://doi.org/10.1590/S0104-42302005000200007

Dunbar, S. B; Reilly, C. M; Gray, R. Higgins, M. K., Culler, S., Brittany Butts, \& Butler, J. (2015) Randomized clinical trial of an integrated self-care intervention for persons with heart failure and diabetes: quality of life and physical functioning outcomes, J. Card. Fail. 10.1016/j.cardfail.2015.05.012

Feijó, M. K. E. F., \& Biolo, A., Silva, E. R. R. (2013 Adaptação e aplicabilidade de um algoritmo de diurético para pacientes com insuficiência cardíaca. Arq. Bras. Cardiol. doi.org/10.5935/abc. 20130089

Folstein, M. F., Folstein, S. E., \& Mchugh, P. R (1975). Mini-Mental State: a practical method for grading the cognitive state of patients for clinician. J Psychiatr Res. 10.1016/0022-3956(75)90026-6.

Flores, P. V. P., Rocha, P. A., Figueiredo, L. S., Guimarães, T. M. L; Velasco, N. S., \& Cavalcanti, A. C. D. (2020) Efeito da entrevista motivacional no autocuidado do paciente com insuficiência cardíaca: ensaio clínico randomizado. Rev. esc. enferm. USP. http://dx.doi.org/10.1590/s1980-220x2019013703634.

Harikrishnan, S., Jeemon, P., Ganapathi, S., Agarwal, A., Viswanathan, S., Sreedharan, M., \& Huffman, M. D. (2021). Five-year mortality and readmission rates in patients with heart failure in India: Results from the Trivandrum heart failure registry. 10.1016/j.ijcard.2020.10.012.

Hua, C. Y., Huang, Y. H., Su, J. Y., Bu, H., \& Tao, M. (2017) Collaborative care model improves self-care ability, quality of life and cardiac function of patients with chronic heart failure. Brazilian Journal Of Medical And Biological Research. 10.1590/1414-431X20176355.

Koirala, B., Himmelfarb, C. D., Budhathoki, C., Tankumpuan, T., Asano, R., \& Davidson, P. M. (2018) Factors affecting heart failure self-care: An integrative review. Heart \& Lung: The Journal of Cardiopulmonary and Acute Care. https: //doi.org/10.1016/j.hrtlng.2018.09.004

Lenardt, M. H., Michel, T., Wachholz, P. A., Borghi, A. S., \& Seima, M. D. (2009). O desempenho de idosas institucionalizadas no miniexame do estado mental. Acta paul. Enferm. doi.org/10.1590/S0103-21002009000500007.

Linn, A. C., Azzolin, K., \& Souza, E. N. (2016) Associação entre autocuidado e reinternação hospitalar de pacientes com insuficiência cardíaca. Rev. Bras. Enferm. http://dx.doi.org/10.1590/0034-7167.2016690312i 
Mantovani, V. M., Ruschel, K. B., Souza, E. N., Mussi, C., \& Silva, E. R. R. (2015) Adesão ao tratamento em pacientes com insuficiência cardíaca que recebem visitas domiciliares com assistência de enfermagem. Acta Paul Enferm. 10.1590/1982-0194201500008.

Mota, F. G. A., Menezes, B. C., Bezerra, B. S., Queiroz, D. T., Carneiro, D. T. O., Barros, F. O., Mota, J. V. F., \& Lima, W. P. M. (2020) Diagnósticos de Enfermagem no paciente com insuficiência cardíaca: Estudo de caso. Braz. J. Hea. Rev. 10.34119/bjhrv3n5-132

Montera, M. W., Almeida, R. A., Tinoco, E. M., Rocha, R. M., Moura, L. Z., Réa-Neto, A., et al. Sociedade Brasileira de Cardiologia. II Diretriz Brasileira de Insuficiência Cardíaca Aguda.Arq Bras Cardiol.2009;93(3 supl.3):1-65

Pereira F. A. C. (2003). O Autocuidado na Insuficiência Cardíaca: tradução, adaptação e validação da european heart failure self-care behaviour scale para o contexto português [dissertação]. Porto: Instituto de Ciências Biomédicas Abel Salazar da Universidade do Porto em Ciências de Enfermagem.

Qavam, S. M., Sahebi, A, Shohani, M., Balavandi, F., Qavam, R., \& Tavan, H. (2017). Investigating the effect of education on self-care among chronic heart failure patients admitted to Shahid Mostafa Khomeini Hospital of Ilam. Glob J Health Sci. 10.5539/gjhs.v9n5p79.

Rector, T. S., Conh, J. N. (1992). Assessment of patient outcome with the Minnesota Living With Heart Failure Questionnaire: reliability and validity during a randomized, double-blind, placebo-controlled trial of pimobendan. Am Heart J. American Heart Journal. doi.org/10.1016/0002-8703(92)90986-6

Riegel, B., Lee, C. S., Dickson, V. V., \& Carlson, B. (2009). An update on the self-care heart failure index. J Cardiovasc Nurs. 10.1097/JCN.0b013e3181b4baa0.

Rohde, L. E., Silva, L. B., Goldraich, L., Grazziotin, T. C., Palombini, D. V., Polanczyk, C. A., \& Clausell, N. (2004). Reliability and prognostic value of traditional signs and symptoms in outpatients with congestive heart failure. Can J Cardiol.

Sauer, J., Rabelo, E. R., Castro, R. A., Goldraich, L., Rohde, L. E., Clausell, N., \& Silva, L. B. (2010). Nurses performance in classifying heart failure patients based on physical exam: comparison with cardiologist's physical exam and levels of N-terminal pro-B-type natriuretic peptide. J Clin Nurs. 10.1111/j.13652702.2010.03403.x.

Simão, A. F., Précoma, D. B., Andrade, J. P. D., Correa, H., Saraiva, J. F. K., Oliveira, G. M. M., \& Souza, W. K. S. B. (2013). I Diretriz brasileira de prevenção cardiovascular. Arquivos brasileiros de cardiologia, 101, 1-63.

Srisuk, N., Cameron, J., Ski, C. F., \& Thompson, D. R. (2016). Randomized Controlled Trial Of Family-Based Education For Patients With Heart Failure And Their Carers. Journal Of Advanced Nursing. 10.111

Sousa, M. M., Oliveira, J. S., Soares, M. J. G. O., \& Bezerra, S. M. S. (2017) Efeitos físicos e psicossociais da insuficiência cardíaca na percepção da qualidade de vida. Cogitare Enfermagem. http://dx.doi.org/10.5380/ce.v22i2.49783

Stevens, B., Pezzullo, L., Verdian, L., Tomlinson, J., George, A., \& Bacal, F. (2018). The economic burden of heart conditions in Brazil. Arquivos brasileiros de cardiologia, 111, 29-36. https://doi.org/10.5935/abc.20180104

Takeda, A., Martin, N., Taylor, R. S., \& Taylor, S. J (2019). Intervenções de gerenciamento de doenças para insuficiência cardíaca. Cochrane Database of Systematic Reviews. doi.org/10.1002/14651858.CD002752.pub4

Timby, B. K. Conceitos e habilidades fundamentais no atendimento de enfermagem. Tradução: UNICOVSKY, Margarita Ana Rubin. (8a ed.), Artmed, 2007.

The WHOQOL Group. The World Health Organization. quality of life assessment (WHOQOL): position paper from the World Health Organization. Soc Sci Med. 199541(10):1403-10. http://www.sciencedirect.com/science/article/pii/027795369500112K.

Tsanani, S.E., Goloyner, M., Ohad, M., Mordechai, D., Sade, Z., Iktelat, A., Rothman, J., \& Oppenheimer, Y. (2016). Evaluation of telehealth service for patients with congestive heart failure in the north of Israel. Eur J Cardiovasc Nurs. 10.1177/1474515115602677.

Oliveira, J. A. D., Cordeiro, R. G., Rocha, R. G., Guimarães, T. C. F., \& Albuquerque, D. C. D. (2017). Impact of telephone monitoring on patients with heart failure: a randomized clinical trial. Acta Paulista de Enfermagem. doi.org/10.1590/1982-0194201700050

Urbich, M., Globe, G., Pantiri, K., Heisen, M., Bennison, C., Wirtz, H. S., \& Di Tanna, G. L. (2020). A systematic review of medical costs associated with heart failure in the USA (2014-2020). PharmacoEconomics, 1-18. 10.1007/s40273-020-00952-0.

Whitaker-Brown, C. D., Woods, S. J., Cornelius, J. B., Southard, E, \& Gulati, S. K. (2017). Improving quality of life and decreasing readmissions in heart failure patients in a multidisciplinary transition-to-care clinic. Heart Lung. 10.1016/j.hrtlng.2016.11.003 